This is an author-created, un-copyedited version of an article accepted for publication in 2D Materials. The publisher is not responsible for any errors or omissions in this version of the manuscript or any version derived from it. The Version of Record is available online at http://dx.doi.org/10.1088/2053-1583/1/3/035004.

The Hall coefficient: a tool for characterizing graphene field effect transistors Peter Wehrfritz and Thomas Seyller

2 D Materials 1 (2014) 035004 


\title{
The Hall Coefficient: A Tool For Characterizing Graphene Field Effect Transistors
}

\author{
Peter Wehrfritz and Thomas Seyller \\ Institut für Physik, Technische Universität Chemnitz, Reichenhainer Str. 70, 09126 \\ Chemnitz, Germany \\ E-mail: thomas.seyller@physik.tu-chemnitz.de
}

Submitted to: 2D Mater.

\begin{abstract}
.
Graphene field effect transistors (GFETs) are considered as a candidate for future high-frequency applications. For their realization, the optimal combination of substrate, graphene preparation, and insulator deposition and composition is required. This optimization must be based on an in-depth characterization of the obtained graphene insulator metal (GIM) stack. Hall effect measurements are frequently employed to study such systems, thereby focussing primarily on the charge carrier mobility. In this work we show how an analysis of the sheet Hall coefficient can reveal further important properties of the GIM stack, like, e.g., the interface trap density and the spacial charge inhomogeneity. To that end, we provide an extensive description of the GIM diode, which leads to an accurate calculation of the sheet Hall coefficient dependent on temperature and gate voltage. The gate dependent inverse sheet Hall coefficient is discussed in detail before we introduce the concept of an equivalent temperature, which is a measure of the spacial charge inhomogeneity. In order to test the concept, we apply it to evaluate already measured Hall data taken from the literature. This evaluation allows us to determine the Drude mobility, even at the charge neutrality point, which is inaccessible with a simple one band Hall mobility analysis, and to shed light on the spacial charge inhomogeneity. The formalism is easily adaptable and provides experimentalists a powerful tool for the characterization of their graphene field effect devices.
\end{abstract}




\section{Introduction}

For ten years now, since Novoselov et al [1] reported on an electric field effect in atomically thin carbon layers, graphene is considered an outstanding new material for future electronic devices. Owing to the absence of an electronic band gap, it is today clear that graphene is not a serious competitor for silicon in logical circuits [2], at least with the conventional horizontal device design. On the other hand, graphene has been demonstrated to be a remarkable rival $[3,4,5,6]$ for analog $\mathrm{RF}$ circuits. The core of such circuits is formed by graphene field effect transistors (GFETs), whose performance is well described by the graphene insulator metal (GIM) diode as discussed below. In many cases, especially for the frequently employed $300 \mathrm{~nm}$ thick silicon dioxide insulator layer, the GIM diode is treated as an ordinary metal plate capacitor. This approximation is proper - in the absence of interface traps - for thick insulating layers, as we will demonstrate later. However, for thin dielectrics or in the presences of interface traps this approach becomes inadequate.

For real applications, devices with a small gate voltage and, therefore, large gate capacitance are required. Here the plate capacitor approximation fails because of the differential capacitance of the graphene channel, which is also known as quantum capacitance (QC). The name QC is inherited from the differential capacitance of traditional 2D electron gases (2DEG). Unlike for 2DEGs, the QC of graphene is not a constant or quantized in any way. It is even dependent on the temperature. Although the name $\mathrm{QC}$ is perhaps misleading, we use it henceforth. The potential dependence of the QC makes it more difficult to find an expression that relates the applied gate voltage to the actual Fermi level position.

There already exists some theoretical understanding of different aspects of GFET devices. The main description of the GIM diode can be borrowed from the theory of metal insulator semiconductor diodes (MIS) [7, 8]. The QC was derived by Fang et al [9] and the temperature dependent charge carrier concentration was explained by Ristein et al [10]. Charge inhomogeneities, also known as charge puddles, have been considered by Zhu et al [11] and interface traps were discussed in a detailed concept by Takase et al [12]. Finally, the non linear $I-V$ characteristics and the current saturation-like behavior [13] was explained by Thiele et al [14] and Champlain [15]. On the other hand, Hall effect measurements are frequently carried out as a means of characterizing graphene field effect devices. Hence, it is worth studying how the combination of the above mentioned effects influences the Hall sheet resistance of these devices. This is the topic of the present work.

The paper is organized as follows. First we consider the charge carrier concentration in neutral and doped graphene. Then we explain the fundamentals of the GIM diode, in a similar manner as Champlain [15], in order to introduce a method which enables the correlation of surface potential and gate voltage. Next, we present a detailed discussion and explanation of the main features of the gate dependent (inverse) sheet Hall coefficient and how it is influenced by temperature, quantum capacitance 
and interface traps. In this context we will introduce the concept of an equivalent temperature, which is a measure of the device homogeneity. Finally, we will model the data of actually measured devices and discuss the concept.

\section{Charge carrier concentration}

The band structure of graphene is linear in the important range near the Dirac point [16] and the resulting density of states is also linear [17]

$$
D(E)=\frac{2}{\pi \hbar^{2} v_{\mathrm{F}}^{2}}\left|E-E_{\mathrm{D}}\right|=\kappa\left|E-E_{\mathrm{D}}\right|
$$

with the Fermi velocity $v_{\mathrm{F}}$, the proportionality constant $\kappa=1.18 \times 10^{14} \mathrm{~cm}^{-2}(\mathrm{eV})^{-2}[18$, $10]$, and $E_{\mathrm{D}}$ the energy of the Dirac point. By equation (1) the electron concentration $n$ and the hole concentration $p$ can be calculated by the convolution of $D(E)$ with the Fermi-Dirac distribution $f(E)$. In figure 1 the density of states and the density of occupied states $D(E) f(E)$ is displayed for n-type and charge neutral graphene. At a temperature $T=0$ the Fermi-Dirac distribution becomes the Heaviside function (see figure $1(\mathrm{a})$ ). Every state below $E_{\mathrm{F}}$ is occupied and every state above $E_{\mathrm{F}}$ is unfilled. At finite temperatures a few states above $E_{\mathrm{F}}$ are occupied due to the thermally excited electrons leaving unfilled states below $E_{\mathrm{F}}$ as can be seen in figure 1(b). Even at charge neutrality (figure 1(c)), a finite number of thermally excited electrons and holes exists. Consequently, the charge carrier concentration $c=n+p$ does not become zero for any conceivable doping.

For a quantitative discussion one has to solve the integral over $f(E) D(E)$, which was already done by Ristein et al [10]. For the electron concentration the result is

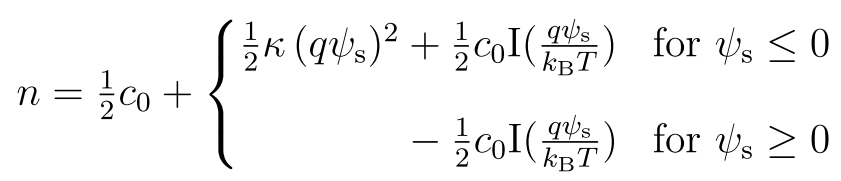

with the intrinsic charge carrier concentration

$$
c_{0}=\frac{\pi^{2}}{6} \kappa\left(k_{\mathrm{B}} T\right)^{2}
$$

the positive elemental charge $q$, the surface potential $\psi_{\mathrm{s}}=\frac{1}{q}\left(E_{\mathrm{D}}-E_{\mathrm{F}}\right)$, the temperature $T$, the Boltzmann constant $k_{\mathrm{B}}$, and the integral

$$
\mathrm{I}(x)=\frac{12}{\pi^{2}} \int_{0}^{|x|} \ln \left(1+\mathrm{e}^{-t}\right) d t .
$$

The function $\mathrm{I}(x)$ has an upper limit of $\lim _{x \rightarrow \pm \infty} \mathrm{I}(x)=1$ ensuring that for high p-type doping $n$ goes to zero [10]. The integral is easily computed numerically (an example Igor Pro implementation can be found in the supplementary information) and is shown in figure $2(\mathrm{a})$. The function $\mathrm{I}(x)$ rapidly converge to 1 . Already with $q \psi_{\mathrm{s}}=4 k_{\mathrm{B}} T$, the deviation to its limit is only $2.2 \%$. 
(a)
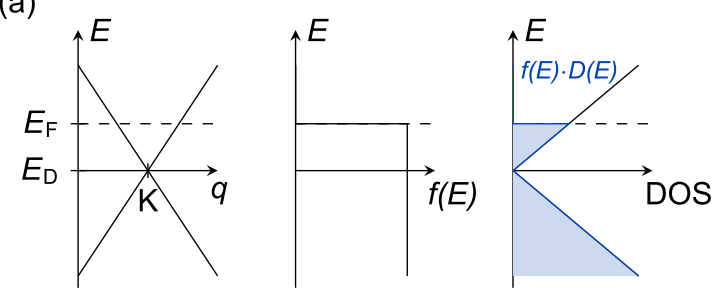

(b)
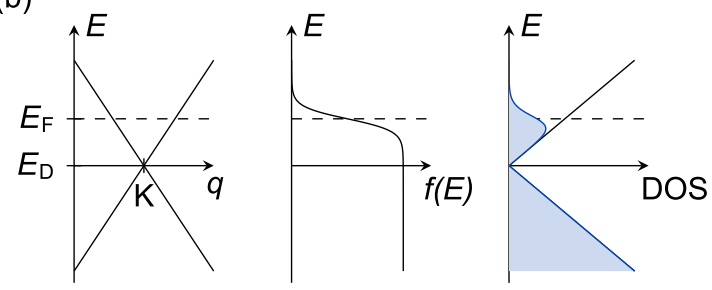

(c)
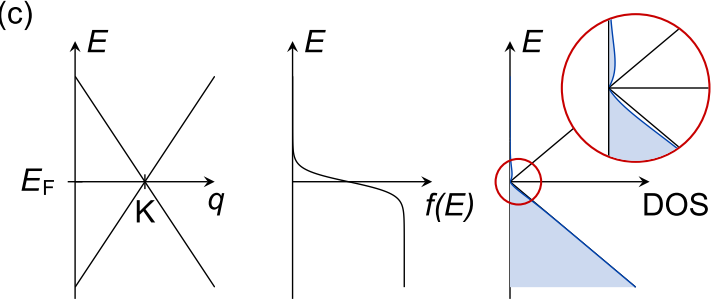

Figure 1. Diagram of the band structure of graphene near the K point (left), the Fermi distribution (center), and the density of occupied states (right) for n-type $\left(E_{\mathrm{F}}-E_{\mathrm{D}}=0.2 \mathrm{eV}\right)$ doped graphene at $(\mathrm{a}) 0 \mathrm{~K}$ and $(\mathrm{b})$ room temperature, and for (c) neutral graphene $\left(E_{\mathrm{F}}=E_{\mathrm{D}}\right)$ at room temperature.

Due to the symmetric density of states the hole concentration can be calculated with $p\left(\psi_{\mathrm{s}}\right)=n\left(-\psi_{\mathrm{s}}\right)[10]$. The sum of them

$$
c \equiv n+p=c_{0}+\frac{1}{2} \kappa\left(q \psi_{\mathrm{s}}\right)^{2}
$$

is the charge carrier concentration, where $c_{0}$ is the temperature dependent intrinsic charge carrier concentration defined in equation (3), which values $1.3 \times 10^{11} \mathrm{~cm}^{-2}$ at room temperature. The charge carrier concentration is always greater or equal zero.

Another useful term is the net charge density

$$
Q_{\mathrm{N}} \equiv q(p-n)=q \operatorname{sgn}\left(\psi_{\mathrm{s}}\right)\left[\frac{1}{2} \kappa\left(q \psi_{\mathrm{s}}\right)^{2}+c_{0} \mathrm{I}\left(\frac{q \psi_{\mathrm{s}}}{k_{\mathrm{B}} T}\right)\right] .
$$

In the case of $\left|q \psi_{\mathrm{s}}\right| \gg k_{\mathrm{B}} T$, the absolute net charge density $\left|Q_{\mathrm{N}}\right|$ approaches $q c$. Only at $T=0,\left|Q_{\mathrm{N}}\right|$ is equal to $q c$ for every value of $\psi_{\mathrm{s}}$. A comparison of $q c$ and $Q_{\mathrm{N}}$ as a function of $\psi_{\mathrm{s}}$ is presented in figure 2 for room temperature and $T=0$.

\section{GIM-Diode}

The graphene insulator metal (GIM) diode forms the core of GFETs. This two terminal device consists of a graphene sheet, a metal electrode, and an insulating layer in between. In the first approximation, it behaves like a conventional metal plate capacitor. By applying a voltage between the metal electrode and the graphene layer, charge will be 


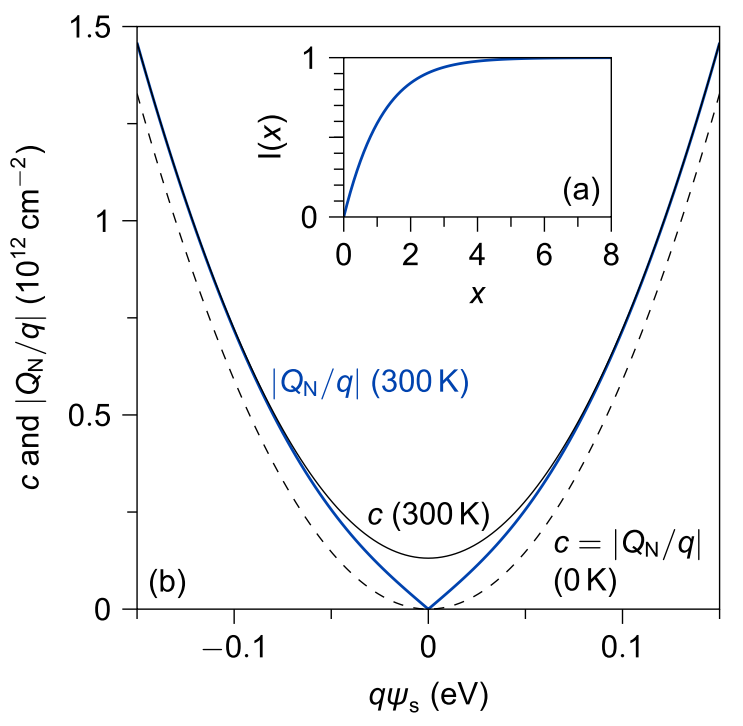

Figure 2. (a) The function $\mathrm{I}(x)$. (b) Comparison of $q c$ and $Q_{\mathrm{N}}$ at room temperature and $T=0$.

(a)

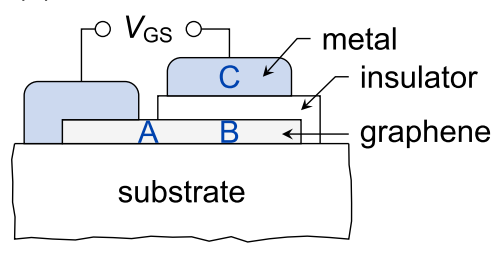

(b)

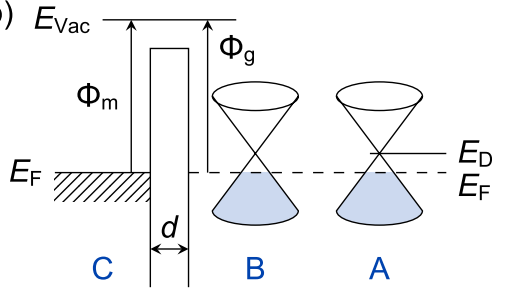

(c)

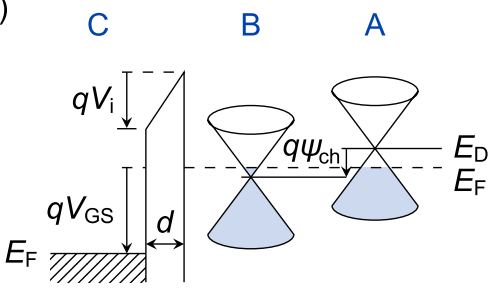

Figure 3. The GIM diode: (a) schematic device layout, (b) band diagram of an initially p-type doped graphene layer for the short circuit $\left(V_{\mathrm{GS}}=0\right)$ case, and (c) with an applied positive voltage. (b and c: based on Sze [7], but adapted to graphene)

transfered from the metal to the graphene layer. But unlike a metal plate capacitor, the capacitance of such a graphene based device is a function of the applied voltage $V_{\mathrm{GS}}$. That is a consequence of the QC as detailed below.

Figure 3(a) shows such a device. Note, that there is an intentional gap (labeled with A) between the gated region and the graphene metal contact. Technically this is not needed or even wanted, but the surface potential of this uncovered area will serve as a reference potential in the following discussion, similar to the bulk potential in MIS diodes [7]. For convenience we will first consider the ideal case: the work function of the metal electrode $\Phi_{\mathrm{m}}$ matches the work function of the graphene layer $\Phi_{\mathrm{g}}$ and we neglect insulator charges and interface traps. The band diagram for the short circuit condition is shown in figure 3(b) here for an initially p-type doped graphene layer. The metal electrode (labeled with $\mathrm{C}$ ) and the graphene share a common Fermi level due to the connection. If now a positive voltage is applied, electrons from the metal electrode will flow to the graphene layer (labeled B in figure 3) directly underneath the metal electrode. Once the system is in equilibrium, the graphene electrode (B) and the 
reference point (A) will have a common Fermi level. Because of the additional charge in the graphene electrode, the surface potential is changed and consequently there is a potential difference $\psi_{\text {ch }}$ between the reference point and the graphene electrode. The rest of the applied voltage drops over the insulating layer $\left(V_{\mathrm{i}}\right)$, so that

$$
V_{\mathrm{GS}}=V_{\mathrm{i}}+\psi_{\mathrm{ch}}
$$

The potential drop $\psi_{\mathrm{ch}}$ is connected with the surface potential via

$$
\psi_{\mathrm{s}}=\psi_{0}-\psi_{\mathrm{ch}}
$$

where $\psi_{0}$ denotes the initial doping, or in other terms $\psi_{0}$ is the surface potential of the reference point.

The equivalent circuit of the diode consists of two series capacitors resulting in a total capacitance per unit area $C$, which meets the condition

$$
\frac{1}{C}=\frac{1}{C_{\mathrm{i}}}+\frac{1}{C_{\mathrm{g}}}
$$

with $C_{\mathrm{i}}$ (sometimes called $C_{\mathrm{ox}}$ ) being the capacitance per unit area of the insulating layer and $C_{\mathrm{g}}$ that of the graphene sheet, which is the QC. It is defined as [7]:

$$
C_{\mathrm{g}} \equiv \frac{\partial}{\partial \psi_{\mathrm{ch}}} Q_{\mathrm{g}}\left(\psi_{0}-\psi_{\mathrm{ch}}\right)=\frac{\partial}{\partial \psi_{\mathrm{s}}} Q_{\mathrm{N}}\left(\psi_{\mathrm{s}}\right)
$$

where $Q_{\mathrm{g}}$ is the additional charge density on the graphene layer due to the applied gate voltage. With equation (6) one gets [10]

$$
C_{\mathrm{g}}=q^{2} \kappa\left[2 k_{\mathrm{B}} T \ln \left(1+\mathrm{e}^{-\frac{q \psi_{\mathrm{s}}}{k_{\mathrm{B}} T}}\right)+q \psi_{\mathrm{s}}\right]
$$

which is mathematically equivalent to the formula derived by Fang et al [9].

With equation (9) and (11), it is possible to calculate the total capacitance per unit area as function of the surface potential. To compare it with actual measurements one has to know the relationship between surface potential and the applied voltage, i.e., $\psi_{\mathrm{s}}\left(V_{\mathrm{GS}}\right)$. This relationship was measured by Kelvin probe microscopy [19], but can also be calculated as shown in the following. The sum of the excess charge density on the metal electrode $Q_{\mathrm{m}}=-C_{\mathrm{i}} V_{\mathrm{i}}$ and $Q_{\mathrm{g}}$ must be zero due to charge conservation, then we get

$$
C_{\mathrm{i}} V_{\mathrm{i}}=\int_{\psi_{\mathrm{s}}}^{\psi_{0}} C_{\mathrm{g}}(\psi) d \psi
$$

Note, that because $C_{\mathrm{g}}$ is not a constant, we have to integrate over $C_{\mathrm{g}}$. Unfortunately, in some publications $[20,11]$ this integration is omitted in favor of an ordinary product resulting in a mismatch by a factor of 2 [14]. The integral of $C_{\mathrm{g}}$ is obviously the net charge density $Q_{\mathrm{N}}$. With equation (7) and (8) we can write

$$
0=C_{\mathrm{i}} \cdot\left(V_{\mathrm{GS}}+\psi_{\mathrm{s}}-\psi_{0}\right)+Q_{\mathrm{N}}\left(\psi_{\mathrm{s}}\right)-Q_{\mathrm{N}}\left(\psi_{0}\right) \equiv g\left(\psi_{\mathrm{s}}\right) .
$$

This expression gives an implicit connection between $\psi_{\mathrm{s}}$ and $V_{\mathrm{GS}}$. For any given $V_{\mathrm{GS}}$, it is not difficult to find the root of $g\left(\psi_{\mathrm{s}}\right)$ numerically. 

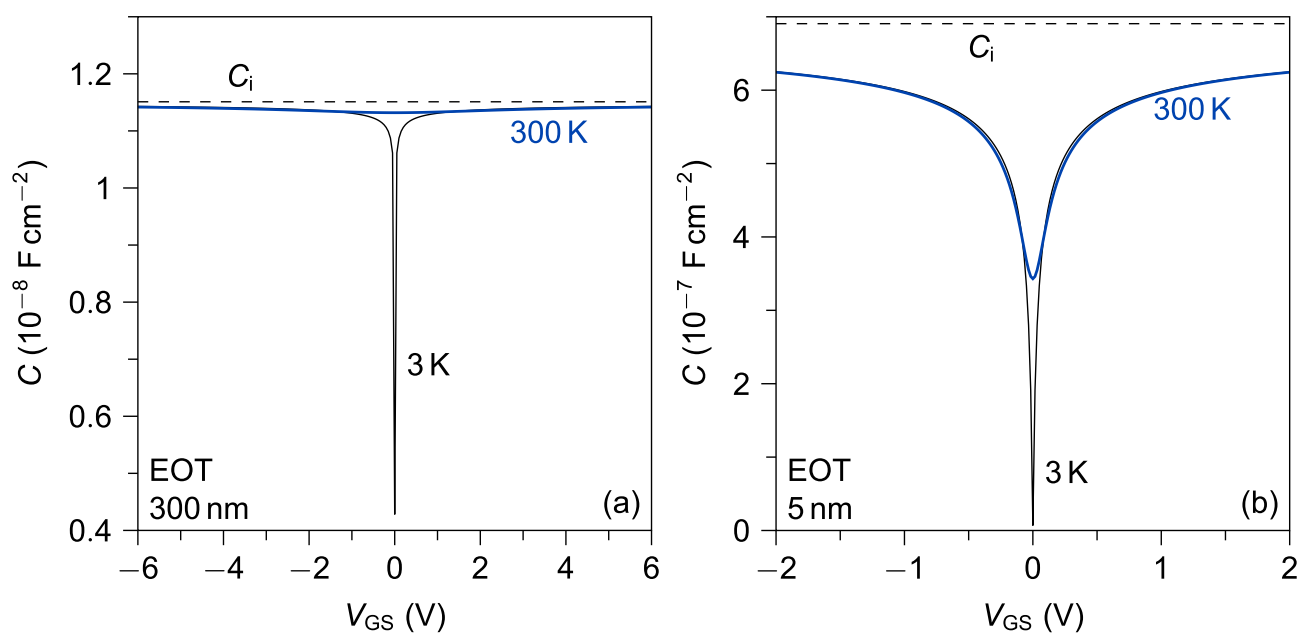

Figure 4. The calculated total capacitance per unit area $C$ of GIM diode as a function of the applied voltage $V_{\mathrm{GS}}$ at $3 \mathrm{~K}$ and $300 \mathrm{~K}$ for an EOT of (a) $300 \mathrm{~nm}$ and (b) $5 \mathrm{~nm}$.

The procedure we use to calculate for example $C\left(V_{\mathrm{GS}}\right)$ works in the following way. First, the surface potential $\psi_{\mathrm{s}}$ is computed for the given $V_{\mathrm{GS}}$. This is done by finding the root of $g\left(\psi_{\mathrm{s}}\right)$ given by equation (13) numerically. Many scientific programming environments offer a build-in method for such a task. The parameters $\psi_{0}, T$, and $C_{\mathrm{i}}$ are determined by the investigated system. Then, using this value for $\psi_{\mathrm{s}}$, the total capacitance per unit area is evaluated. Actually, this method allows the calculation of other quantities as well such as $c\left(\psi_{\mathrm{s}}\right), Q_{\mathrm{N}}\left(\psi_{\mathrm{s}}\right), n\left(\psi_{\mathrm{s}}\right) p\left(\psi_{\mathrm{s}}\right)$, and $C_{\mathrm{g}}\left(\psi_{\mathrm{s}}\right)$ as function of the applied voltage $V_{\mathrm{GS}}$. Figure 4 shows the calculated total capacitances per unit area for a device with a large equivalent oxide thickness (EOT) of $300 \mathrm{~nm}$ and one with a small EOT of $5 \mathrm{~nm}$ at low temperature $(3 \mathrm{~K})$ and room temperature. The initial doping was set to zero $\left(\psi_{0}=0\right)$. With an EOT of $300 \mathrm{~nm}$, which is usually used for back gated exfoliated graphene [21, 22], the capacitance per unit area is almost constant and differs only slightly $(<1.6 \%)$ from $C_{\mathrm{i}}$. Even at low temperature, there is only a very narrow region, near the neutrality point, where the effect is significant. In practice this is hard to measure since already small inhomogeneities in the spatial doping distribution will cancel this effect. This small influence is explained by the relatively small $C_{\mathrm{i}}$ compared to the QC. Only near the charge neutrality, they are of the same order of magnitude. Consequently, the frequently used approximation for the net charge density

$$
Q_{\mathrm{g}} \approx C_{\mathrm{i}} V_{\mathrm{GS}}
$$

is here reasonable in the case of a thick insulator.

A different picture emerges with an EOT of $5 \mathrm{~nm}$. Here (see figure 4(b)), the total capacitance per unit area is significantly lowered in a wide range of $V_{\mathrm{GS}}$ compared to the insulator capacitance $\left(20 \%\right.$ at $V_{\mathrm{GS}}=0.5 \mathrm{~V}, 10 \%$ at $\left.V_{\mathrm{GS}}=2 \mathrm{~V}\right)$. At low temperature the total capacitance goes down to almost zero at $V_{\mathrm{GS}}=0$. The approximation in equation (14) can serve here only as a rough estimate. 
(a)

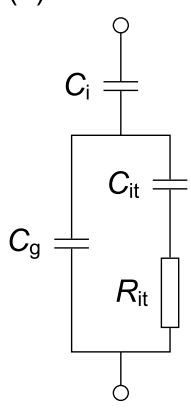

(b)

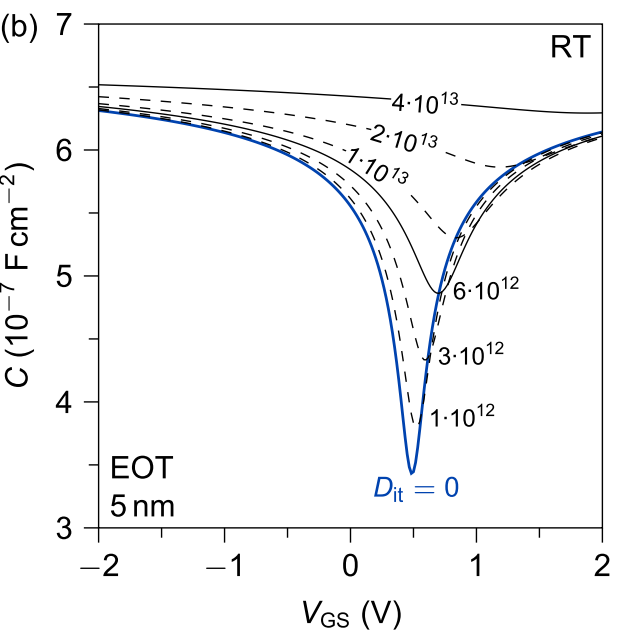

(c)

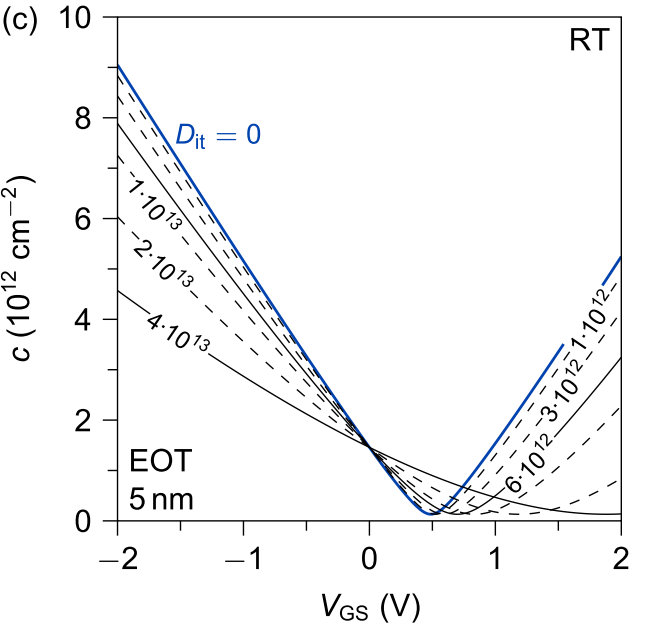

Figure 5. (a) Equivalent circuit of a GIM diode with interface traps (based on Sze [7]). The capacitance per unit area $C$ (b) and the charge carrier concentration $c$ (c) of GIM diodes with a interface trap density $D_{\text {it }}$ of $0 \mathrm{~cm}^{-2} \mathrm{eV}^{-1}, 1 \times 10^{12} \mathrm{~cm}^{-2} \mathrm{eV}^{-1}$, $3 \times 10^{12} \mathrm{~cm}^{-2} \mathrm{eV}^{-1}, \quad 6 \times 10^{12} \mathrm{~cm}^{-2} \mathrm{eV}^{-1}, \quad 1 \times 10^{13} \mathrm{~cm}^{-2} \mathrm{eV}^{-1}, \quad 2 \times 10^{13} \mathrm{~cm}^{-2} \mathrm{eV}^{-1}$ and $4 \times 10^{13} \mathrm{~cm}^{-2} \mathrm{eV}^{-1}$ at $300 \mathrm{~K}$ and an EOT of $5 \mathrm{~nm}$.

\section{Interface traps}

Interface traps, i.e., electrical states at the interface between the semiconductor and the insulator, are known to influence the performance of MIS diodes. The same is true for traps at the interface between graphene and the insulator in the GIM diode. In graphene based systems there can be additional traps at the substrate graphene boundary $[23,10,12]$. By definition, interface traps are electrically connected to the semiconductor or graphene channel [24]. Hence the charge transferred from the metal electrode to the graphene electrode does not only change the net charge of the graphene layer, but a part of the charge is trapped in those interface states. Thus the interface net charge density $Q_{\text {it }}$ depends on the surface potential. The precise relationship is given by the interface trap density $[7,8]$

$$
D_{\mathrm{it}}=\frac{1}{q} \frac{d Q_{\mathrm{it}}}{d E}=\frac{1}{q^{2}} \frac{d Q_{\mathrm{it}}}{d \psi_{\mathrm{s}}}=\frac{1}{q^{2}} \frac{d Q_{\mathrm{it}}}{d \psi_{\mathrm{ch}}}=\frac{1}{q^{2}} C_{\mathrm{it}}
$$

where $C_{\text {it }}$ is the interface trap capacitance per unit area. In general, $D_{\mathrm{it}}$ is not constant, but itself is a function of $\psi_{\mathrm{s}}$ and depends on the type of interface traps. Actually, there is only little literature about interface traps in graphene based systems. Takase et al [12] assumed a constant $D_{\text {it }}$ for a top gated GFET on epitaxial graphene on $\mathrm{SiC}(0001)$. The $D_{\text {it }}$ was valued to $3.7 \times 10^{13} \mathrm{~cm}^{-2} \mathrm{eV}^{-1}$ for the graphene insulator interface and $5.0 \times 10^{12} \mathrm{~cm}^{-2} \mathrm{eV}^{-1}$ for the substrate graphene interface. In contrast, interface trap densities in the order of $10^{10} \mathrm{~cm}^{-2} \mathrm{eV}^{-1}$ can be achieved with traditional MIS diodes [8].

In the equivalent circuit (see figure $5(\mathrm{a})$ ) there is, in parallel to the $\mathrm{QC}$, the interface capacitance per unit area $C_{\text {it }}$ and a resistor $R_{\text {it }}$. Only in the AC regime $R_{\text {it }}$ is important. It dictates how fast charge can be transfered into the interface traps. We only consider 
the static case in this work. To account for the interface traps in the calculation, we have to extend equation (12) with $Q_{\text {it }}$

$$
C_{\mathrm{i}} V_{\mathrm{i}}=\int_{\psi_{\mathrm{s}}}^{\psi_{0}} C_{\mathrm{g}}(\psi)+C_{\mathrm{it}}(\psi) d \psi
$$

In the following calculation we will assume a constant $D_{\text {it }}$. This approximation is reasonable for small interface trap densities because the influence of the traps is small if $C_{\mathrm{g}} \gg C_{\text {it }}$. But if the integral over $D_{\mathrm{it}}\left(\psi_{\mathrm{s}}\right)$ is known and can be calculated either analytically or numerically, it is straightforward to adapt the formalism presented here to handle a non-constant $D_{\mathrm{it}}\left(\psi_{\mathrm{s}}\right)$. Using the same procedure as for equation (13), we can transform equation (16) to

$$
0=C_{\mathrm{i}} \cdot\left(V_{\mathrm{GS}}-\psi_{\mathrm{ch}}\right)-q^{2} D_{\mathrm{it}} \psi_{\mathrm{ch}}+Q_{\mathrm{N}}\left(\psi_{\mathrm{s}}\right)-Q_{\mathrm{N}}\left(\psi_{0}\right) \equiv g_{\mathrm{it}}\left(\psi_{\mathrm{s}}\right) .
$$

With $g_{\text {it }}\left(\psi_{\mathrm{s}}\right)$ we obtained again an implicit correlation of $V_{\mathrm{GS}}$ and $\psi_{\mathrm{s}}$. The solution for $g_{\text {it }}=0$ can be easily calculated numerically. In the supplementary information a sample implementation for Igor Pro can be found.

Figure $5(\mathrm{~b})$ displays $C\left(V_{\mathrm{GS}}\right)$ of GIM diodes with an EOT of $5 \mathrm{~nm}$ for several $D_{\text {it }}$ values. Here the initial doping was set to a value of $\psi_{0}=0.15 \mathrm{~V}$, which corresponds to a charge carrier concentration of $1.5 \times 10^{12} \mathrm{~cm}^{-2}$. Two remarkable features are apparent. First, the minimum of $C\left(V_{\mathrm{GS}}\right)$ shifts to higher voltages as the trap density increases. Second, the ratio of the minimal to maximal value of $C$ drops with increasing $D_{\text {it }}$. With $D_{\text {it }}=1 \times 10^{12} \mathrm{~cm}^{-2} \mathrm{eV}^{-1}$, the difference to the ideal curve is relative small. On the other hand, with $D_{\text {it }}=4 \times 10^{13} \mathrm{~cm}^{-2} \mathrm{eV}^{-1}$, the minimum is hardly seen.

In addition, the charge carrier concentration was calculated in figure 5(c). All devices exhibit the same $c$ at $V_{\mathrm{GS}}=0$. This is not the case if for example insulator charges or other influences cause a non-zero flat band voltage (see section 5). Apparently, the effective capacitance is reduced with increasing interface traps density. With $D_{\text {it }}=$ $1 \times 10^{12} \mathrm{~cm}^{-2} \mathrm{eV}^{-1}$ at $V_{\mathrm{GS}}=-2 \mathrm{~V}$, a charge carrier concentration of $8.8 \times 10^{12} \mathrm{~cm}^{-2}$ can be reached while with $4 \times 10^{13} \mathrm{~cm}^{-2} \mathrm{eV}^{-1}$, only $c=4.6 \times 10^{12} \mathrm{~cm}^{-2}$ is obtained. This corresponds to a degrease of the effective capacitance by a factor of 2.4. Furthermore, the shape of the curve becomes non-linear for high values of $D_{\text {it }}$. This is in good agreement with the experimental observations of Takase et al [12].

\section{Flat Band Voltage}

Another source of a non-ideal behavior of MIS and GIM diodes are mobile or fixed charges in the insulator and work function difference between the metal electrode and graphene. These effects are well studied and understood for conventional MIS diodes. They mainly impact the flat band voltage. The flat band voltage $V_{\mathrm{FB}}$ is the bias which needs to be applied to obtain flat band conditions in the semiconductor when the surface potential equals the bulk potential. While there is "no bulk" in a 2D material, we already defined the reference potential $\psi_{0}$ in a similar manner. Hence for graphene $V_{\mathrm{FB}}$ is the voltage that needs to be applied to have $\psi_{\mathrm{s}}=\psi_{0}$ and thus $\psi_{\mathrm{ch}}=0$. In other words 


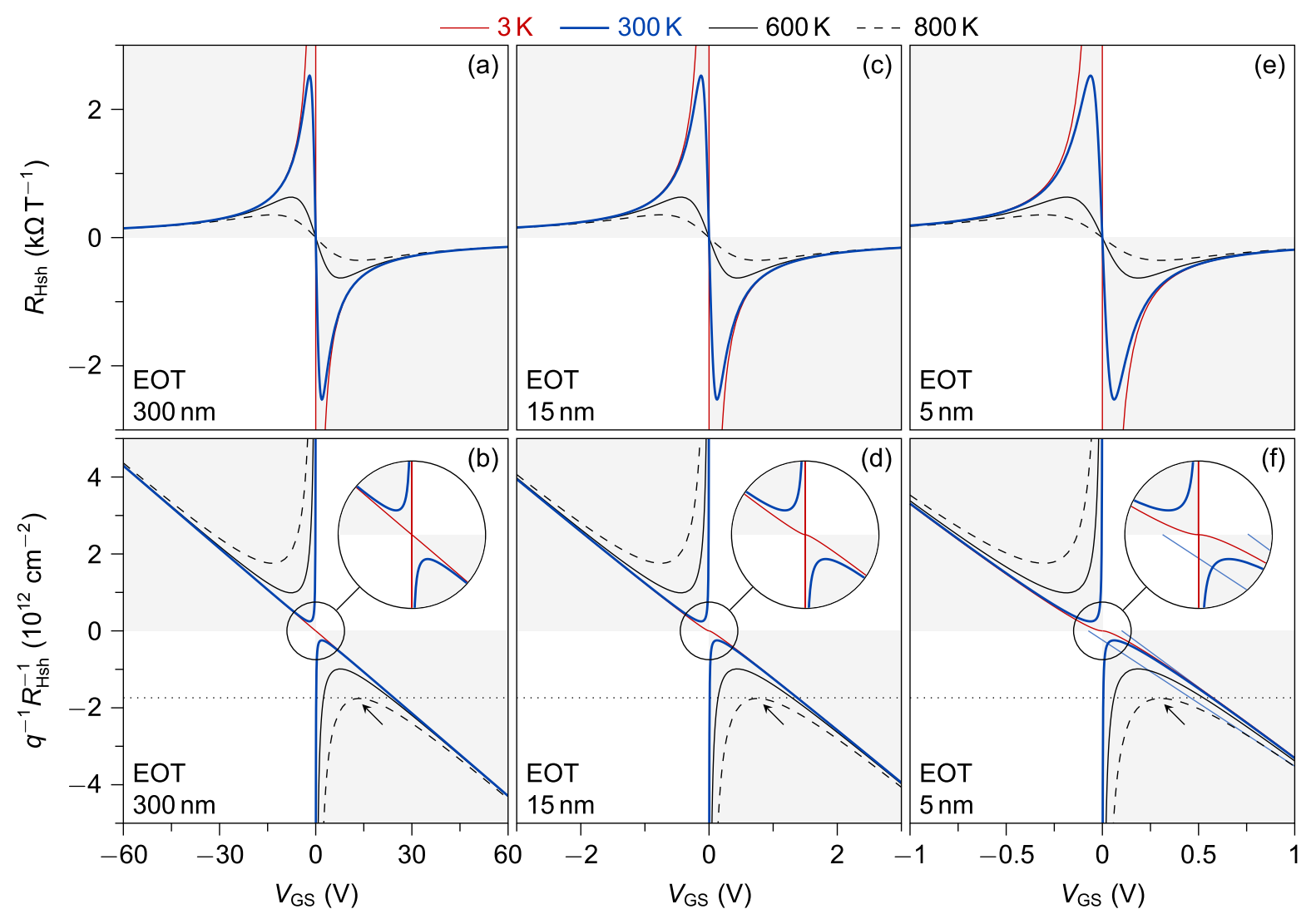

Figure 6. The calculated sheet Hall coefficient of graphene transistors with an EOT of (a) $5 \mathrm{~nm}$, (c) $15 \mathrm{~nm}$ and (e) $300 \mathrm{~nm}$ at several temperatures (solid red line $3 \mathrm{~K}$, solid blue line $300 \mathrm{~K}$, solid black line $600 \mathrm{~K}$ and dashed black line $800 \mathrm{~K}$ ), accordingly the inverse sheet Hall coefficient: (b) $5 \mathrm{~nm}$, (d) $15 \mathrm{~nm}$ and (f) $300 \mathrm{~nm}$.

$V_{\mathrm{FB}}$ is the voltage to be applied to reach the initial doping of the uncovered graphene sheet. With that in mind, it is possible to use the already well-known contributions to $V_{\mathrm{FB}}$ described for example by Sze [7] or Schroder [8]. Basically, the flat band voltage affects a shift in the transfer characteristic and is often loosely attributed to doping by the oxide.

\section{Sheet Hall Coefficient}

A graphene FET device comprises of three terminals: the source and the drain, which are directly connected with the graphene layer and the gate, which controls the charge carrier concentration via $V_{\mathrm{GS}}$. Devices with more terminals, such as Hall bars or van der Pauw test, structures are inapplicable for real high frequency devices, but they are useful for the characterization of the employed graphene insulator metal stack. They permit to measure the sheet resistance $R_{\mathrm{sh}}$ and the sheet Hall coefficient $R_{\mathrm{Hsh}}$.

The sheet Hall coefficient is tightly connected with the electron and hole 
concentration [7]

$$
R_{\mathrm{Hsh}}=r \frac{1}{q} \frac{p-b^{2} n}{(p+b n)^{2}}
$$

with $r$ the Hall scattering ratio and $b$ the ratio of the electron and hole mobility $\mu_{\mathrm{e}} / \mu_{\mathrm{h}}$. For graphene $r=1$ is commonly used, which is in good agreement with experiments [22]. For the sake of simplicity, we will use $b=1$ in the following. This assumption is not generally true, but for the calculation of the sheet Hall coefficient it is justified as a first approximation [11]. Equation (18) then simplifies to

$$
R_{\mathrm{Hsh}}=\frac{1}{q} \frac{p-n}{(p+n)^{2}}=\frac{Q_{\mathrm{N}}}{(q c)^{2}} .
$$

Both the net charge density $Q_{\mathrm{N}}$ and the carrier concentration $c$ were already presented in figure 2 as function of the surface potential.

As we know how to map the applied voltage to the surface potential, it is trivial to calculate the $R_{\mathrm{Hsh}}$ dependent on $V_{\mathrm{GS}}$. Figures 6 shows $R_{\mathrm{Hsh}}$ and its inverse $R_{\mathrm{Hsh}}^{-1}$ at severals temperatures $(T=3 \mathrm{~K}, 300 \mathrm{~K}, 600 \mathrm{~K}$ and $800 \mathrm{~K})$ for devices with EOT of $5 \mathrm{~nm}$, $15 \mathrm{~nm}$ and $300 \mathrm{~nm}$. The initial doping was set to zero that is $\psi_{0}=0$. Near the Dirac point, here for small values of $V_{\mathrm{GS}}, R_{\mathrm{Hsh}}^{-1}$ diverges because $Q_{\mathrm{N}}$ becomes small and crosses zero. Only in the limit of $T=0$, the value of $R_{\mathrm{Hsh}}^{-1}$ at charge neutrality is zero. For purpose of clarity, the poles of the curves for $T=3 \mathrm{~K}$ are only displayed in the magnifier inset in figure 6 . Since we are using $b=1$, the resulting curves are point symmetric. In the following, we will only discuss the positive applied voltage range, thus for the electrons as majority charge carriers.

Due to the pole of $R_{\mathrm{Hsh}}^{-1}$ at charge neutrality, the curve exhibits a local maximum (see the arrow in figure 6(b), 6(d) and 6(f)). The value of the maximum does not depend on the insulator EOT, but on $T$. This is obviously the case because $R_{\mathrm{Hsh}}^{-1}\left(\psi_{\mathrm{s}}\right)$ is a function of the surface potential and its maximum value depends only on the temperature. Numerical calculations reveal the following relationship for the absolute maximum value:

$$
Q_{\star}=\left|\max _{\psi_{\mathrm{s}}<0} R_{\mathrm{Hsh}}^{-1}\left(\psi_{\mathrm{s}}\right)\right|=1.883 \cdot q c_{0}
$$

with the intrinsic charge carrier concentration $c_{0}$ given in equation (5). This makes it very easy to find the intrinsic charge carrier concentration from measured $R_{\mathrm{Hsh}}^{-1}$ data. At rather large surface potentials $\psi_{\mathrm{s}}$, the net charge density $\left|Q_{\mathrm{N}}\right| \approx q c$. Hence, we can write in the first approximation

$$
R_{\mathrm{Hsh}}^{-1} \approx C_{\mathrm{eff}} \cdot\left(V_{\mathrm{GS}}-V_{0, \mathrm{n}}\right) .
$$

Note, that $V_{0, \mathrm{n}}$ (or $V_{0, \mathrm{p}}$ for holes as majority charge carriers, respectively) is not necessarily the corresponding voltage for charge neutrality.

For a thick insulator with an EOT of $300 \mathrm{~nm}$, the total capacitance is nearly constant and consequently the linear approximation equation (21) for the inverse Hall coefficient holds at low temperatures virtually in the full range as can be seen in figure 6(b). At higher temperatures, the magnitude of the local maximum of $R_{\mathrm{Hsh}}^{-1}$ increases and $V_{0}$ shifts 
to more negative values. On the other hand, $R_{\mathrm{Hsh}}$ itself has the shape of a hyperbole at $T=0$. At finite temperatures it will nonetheless become zero at charge neutrality. The emerging global maximum is strongly dependent on the temperature and declines with increasing temperature.

Although the total capacitance of a GIM structure with an EOT of $5 \mathrm{~nm}$ is far from being constant, the linear approximation of $R_{\mathrm{Hsh}}^{-1}$ fits surprisingly well for large applied voltages (see figure 6(f)). Near charge neutrality the total capacitance is noticeably reduced and a plateau is formed for very small temperatures. This entails that in the linear approximation $V_{0, \mathrm{n}}$ now becomes positive. In a similar manner as for an EOT of $300 \mathrm{~nm}, V_{0, \mathrm{n}}$ shifts to smaller values with increasing temperature and can even become negative. Those fine details cannot be deduced from the sheet Hall coefficient (figure $6(\mathrm{f}))$ itself. The shape is similar to that of figure 6(a). Only the voltage range and the positions of the maxima is changed for obvious reasons.

An EOT of $5 \mathrm{~nm}$ is technologically challenging and often thicker insulating layers are used. For comparison, $R_{\mathrm{Hsh}}^{-1}$ and $R_{\mathrm{Hsh}}$ for an EOT of $15 \mathrm{~nm}$ are shown in figure $6(\mathrm{c})$ and $6(\mathrm{~d})$. That is the equivalent thickness of an $\mathrm{Al}_{2} \mathrm{O}_{3}$ layer with a real thickness of approximately $35 \mathrm{~nm}$. The plateau in $R_{\mathrm{Hsh}}^{-1}$ for very small temperatures is here less pronounced and $V_{0, \mathrm{n}}$ is smaller, but still positive at room temperature.

In summary for all GIM diodes presented in figure 6, one can say that the temperature causes a shift of $V_{0, \mathrm{n}}$ to smaller values, whereas an increased insulator capacitance, i.e., a smaller EOT, shifts $V_{0, \mathrm{n}}$ to higher values. The maximal value of $R_{\mathrm{Hsh}}$ and the value of the local maximum of $R_{\mathrm{Hsh}}^{-1}$ depends only on the temperature and not on the insulator capacitance.

One missing issue in the previous discussion is the presence of spatial charge inhomogeneities (SCI) first observed by scanning tunneling microscopy [25]. Those can be caused by ripples [26, 27, 28], charged impurities [29, 30] or by fluctuation in the insulator thickness. The shape of the SCI distribution is, however, not known and depends on the processing details of the device. Zhu et al [11], for instance, assumed a rectangular function to simplify the calculations.

The qualitative consequences, however, can be easily understood. At $T=0$, a GIM diode with inhomogeneous charge distribution will be comprised of areas with excess electrons and areas with excess holes, even at net charge neutrality $\left(Q_{\mathrm{N}}=0\right)$. This results in a charge carrier concentration $c \neq 0$, very similar to what happens when the temperature is increased. Indeed, the measurements of Nagashio et al [31] show that for their devices the differential graphene-channel capacitance $\ddagger$ is hardly dependent on the temperature, presumably due to SCI.

Therefore we propose to use the well-understood temperature dependence of GIM diodes to describe SCI. The equivalent temperature $T_{\text {eq }}$ is then the temperature at which the specific device would reveal the same sheet Hall coefficient in the absence of SCI. In the next section, we will show that it is possible to model $R_{\text {Hsh }}$ with that approach.

$\ddagger$ We intentional do not use the term QC here to make clear that the actual differential graphenechannel capacitance differs from the intrinsic QC in equation (11). 


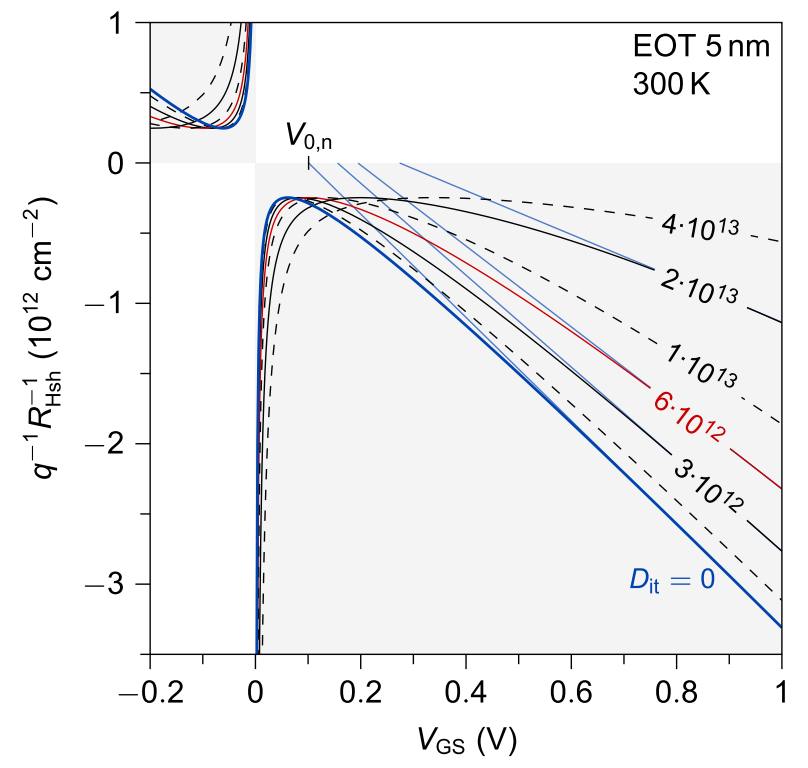

Figure 7. The inverse sheet Hall coefficient for a diode with an EOT of $5 \mathrm{~nm}$ at room temperature with interface traps densities of $0 \mathrm{~cm}^{-2} \mathrm{eV}^{-1}, 1 \times 10^{12} \mathrm{~cm}^{-2} \mathrm{eV}^{-1}$, $3 \times 10^{12} \mathrm{~cm}^{-2} \mathrm{eV}^{-1}, 6 \times 10^{12} \mathrm{~cm}^{-2} \mathrm{eV}^{-1}, 1 \times 10^{13} \mathrm{~cm}^{-2} \mathrm{eV}^{-1}, 2 \times 10^{13} \mathrm{~cm}^{-2} \mathrm{eV}^{-1}$ and $4 \times 10^{13} \mathrm{~cm}^{-2} \mathrm{eV}^{-1}$.

One important point still not discussed, is how interface traps influence the sheet Hall coefficient. With equation (17) we already have demonstrated how to find a term for $\psi_{\mathrm{s}}\left(V_{\mathrm{GS}}\right)$ in the presence of interface traps. Consequently, it is straightforward to calculate the sheet Hall coefficient. In figure $7, R_{\mathrm{Hsh}}^{-1}$ is displayed for several $D_{\text {it }}$ at a temperature of $300 \mathrm{~K}$ for a device with an EOT of $5 \mathrm{~nm}$. As already mentioned, $Q_{\star}$ only depends on the temperature and spatial charge inhomogeneities, hence it is independent of the interface trap density. Due to the reduced effective capacitance in the presence of interface traps, the curves of $R_{\mathrm{Hsh}}^{-1}$ are broadened. Interestingly, the absolute values of $V_{0}$ are increased with larger $D_{\text {it }}$, although the relative variation in the total capacitance (see figure 5) is reduced. The value of $V_{0}$ is, in all four cases presented in figure 7 , larger then the position of the local maximum of $R_{\mathrm{Hsh}}^{-1}$. This is in contrast to a decrease in the insulator capacitance, which would similarly reduce the effective capacitance.

\section{Application}

The procedure described above for the calculation of the sheet Hall coefficient can be utilized as a fitting function to model experimental $R_{\mathrm{Hsh}}$ data. Here we demonstrate this on the basis of already published sheet Hall coefficient measurements [32]. The GFET test device was made with quasi-freestanding graphene on $6 \mathrm{H}-\mathrm{SiC}(0001)$ surface using a nominally $36 \mathrm{~nm}$ thick silicon nitride film as the top gate material. With a dielectric constant of 6.1, an EOT of $23 \mathrm{~nm}$ was achieved.

Figure 8(a) displays the measured data of that device and a fitting curve. The fit was calculated by the two-step procedure explained in section 3 using equation 

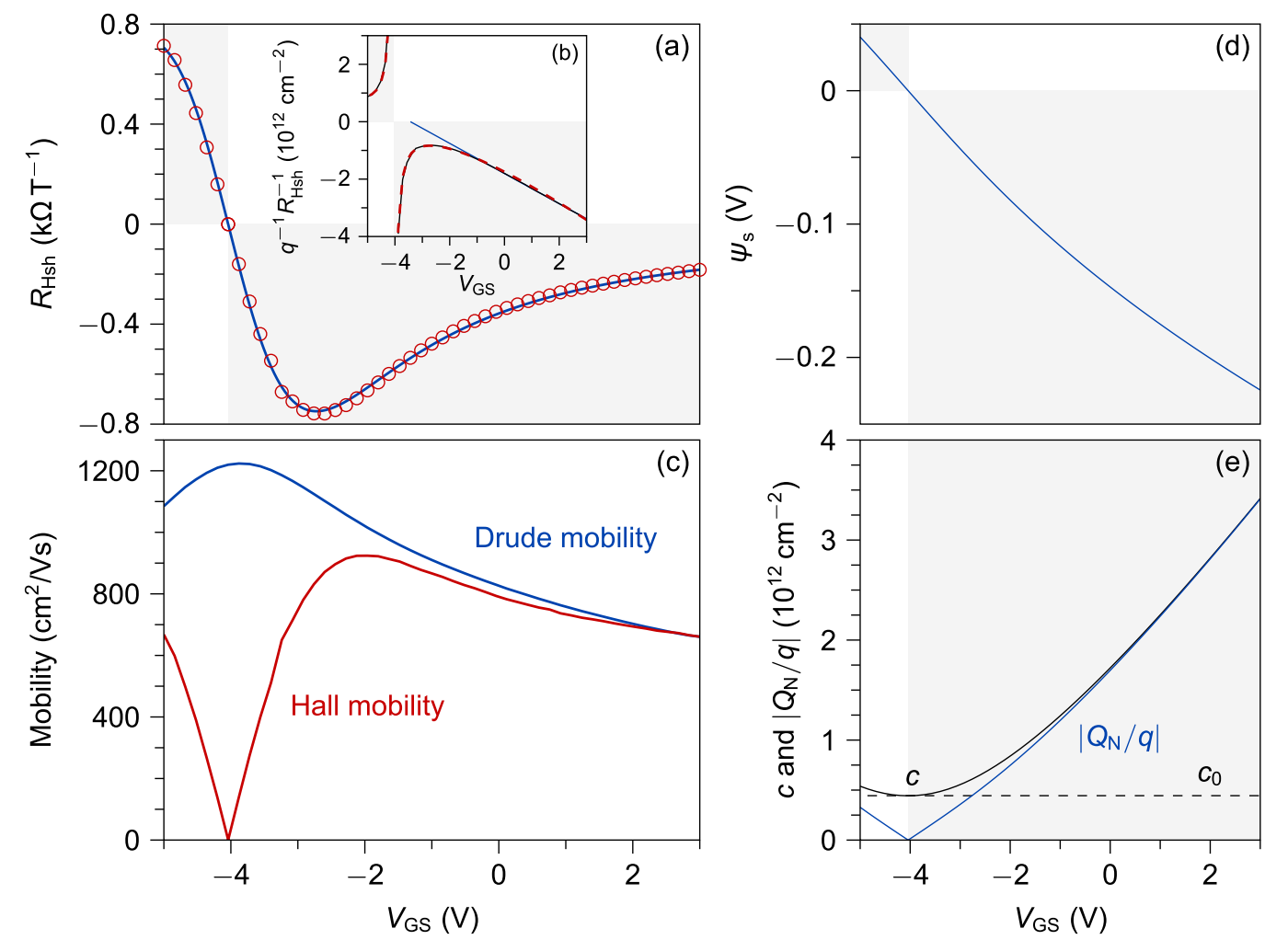

Figure 8. (a) Measured sheet Hall coefficient of top gated epitaxial graphene on $\mathrm{SiC}$ FET test structure (red circles)[32] and the corresponding $R_{\text {Hsh }}$ fitting curve (blue line). Inset (b): The inverse sheet Hall coefficient, measurement (red dashed line) and the fitting curve (blue solid line). (c) Comparison of the Hall and the Drude mobility calculated with equation (22) and (23). (d) The surface potential and (e) the charge carrier concentration and the net charge density as a function of the gate voltage.

(17) and (19). The intermediate results, namely the gate voltage dependence of the surface potential, the charge carrier concentration and the net charge density, are illustrated in figure $8(\mathrm{~d})$ and $8(\mathrm{e})$. The best fit was obtained with $\psi_{0}=-0.147 \mathrm{~V}, D_{\text {it }}=$ $1.3 \times 10^{13} \mathrm{~cm}^{-2} \mathrm{eV}^{-1}$ and an equivalent temperature $T_{\text {eq }}=555 \mathrm{~K}$, which corresponds to a intrinsic charge carrier concentration of $c_{0}=4.44 \times 10^{11} \mathrm{~cm}^{-2}$. This indicates a high, or at least non-ignorable amount of SCI since the measurements were performed at room temperature. For comparison, the Hall data of Novoselov et al [21] can be fitted with an equivalent temperature $T_{\text {eq }}=586 \mathrm{~K}$ (not shown here) although the measurements were performed at $10 \mathrm{~K}$. In figure $8(\mathrm{~b})$, the inverse sheet Hall coefficient is displayed. The linear approximation exposes a positive $V_{0, \mathrm{n}}$ due to the thin insulator.

Besides the equivalent temperature and the interface trap density, we obtained the surface potential as a function of the gate voltage. So we can calculate the charge carrier concentration as a function of $V_{\mathrm{GS}}$. With that gained data, it is possible to not only calculate the Hall mobility [8]

$$
\mu_{\mathrm{H}}=\left|\frac{R_{\mathrm{Hsh}}}{R_{\mathrm{sh}}}\right|
$$




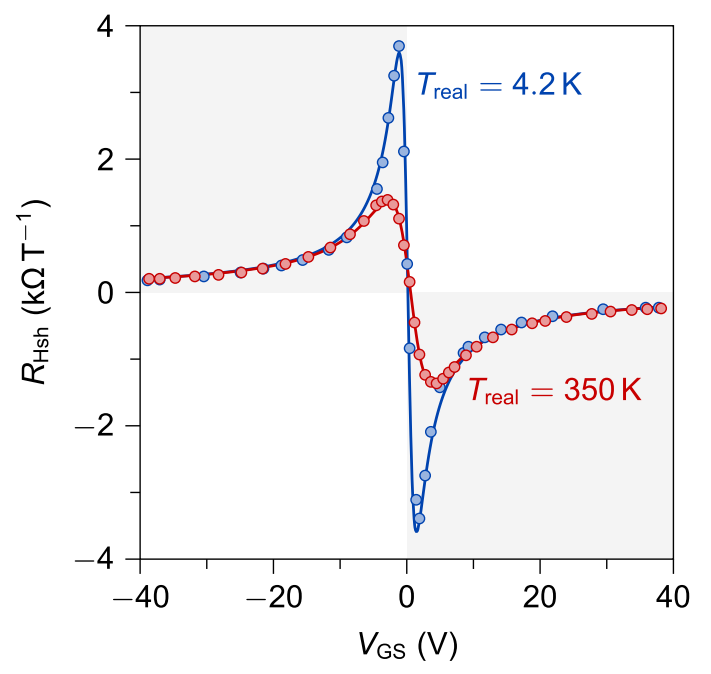

Figure 9. The sheet Hall coefficient of back-gated, exfoliated graphene measured by Zhu et al [11] at temperatures of $4.2 \mathrm{~K}$ and $350 \mathrm{~K}$. The corresponding lines are calculated using the equivalent temperature model.

with the sheet resistance $R_{\mathrm{sh}}$ but also the Drude mobility when we again assume $\mu_{\mathrm{h}}=\mu_{\mathrm{e}}$

$$
\mu_{\mathrm{D}}=\frac{1}{R_{\mathrm{sh}} q c}
$$

Both mobilities are compared in figure 8(c). Near charge neutrality, the Hall mobility becomes small due to the zero-crossing of $R_{\mathrm{Hsh}}$, whereas the Drude mobility approaches its maximum. Note, that the maximum of $\mu_{\mathrm{D}}$ and the minimum of $\mu_{\mathrm{H}}$ are not exactly at the same position indicating that $\mu_{\mathrm{e}}$ and $\mu_{\mathrm{h}}$ are not exactly equal. However, the difference is small and justifies the approximation.

\section{Equivalent Temperature}

The equivalent temperature can serve as a measure of the SCI and can be used to directly compare two devices measured at the same temperature. To extract the influence of the actual temperature, the temperature dependent Hall data of Zhu et al [11] were analyzed in the same way as descried in section 7 . They used exfoliated graphene on $\mathrm{SiO}_{2} / \mathrm{Si}$ with an oxide thickness of $\sim 300 \mathrm{~nm}$. The measured and the corresponding fitted $R_{\mathrm{Hsh}}$ for $T_{\text {real }}=4.2 \mathrm{~K}$ and $350 \mathrm{~K}$ are shown in figure 9 , where $T_{\text {real }}$ denotes the actual temperature in contrast to $T_{\text {eq }}$. For all temperatures, $D_{\text {it }}=0$ and an EOT of $285 \mathrm{~nm}$ was used.

The results of the equivalent temperatures are shown in figure 10. The first important result is the overall smaller $T_{\text {eq }}$, compared to the pioneering work of Novoselov et al [21] four years before. Zhu et al managed to reduced the SCI by more than a factor of 2. The equivalent temperature is not only caused by the SCI, figure 10 shows a clear dependency on $T_{\text {real }}$. Under the assumption of a rectangular SCI distribution, 


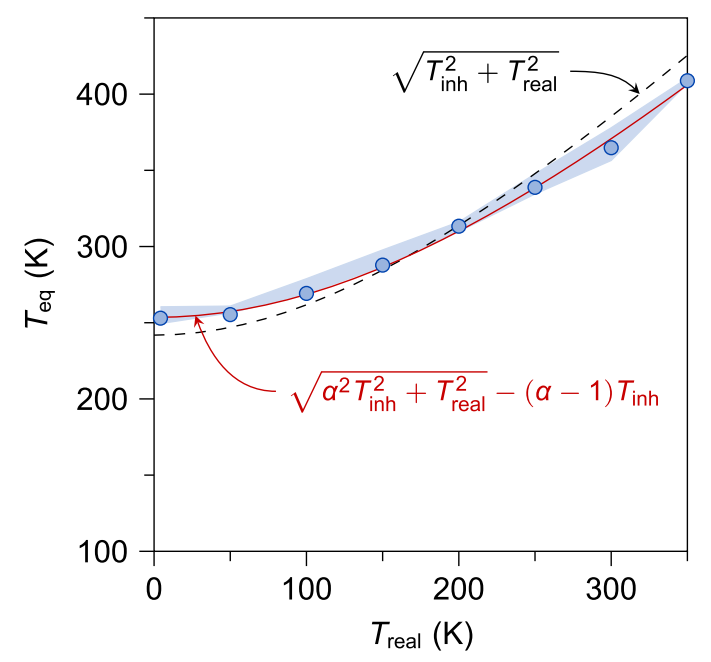

Figure 10. Comparison of the actual temperature $T_{\text {real }}$ and the equivalent temperature $T_{\text {eq }}$ obtained by the evaluation of the Hall measurements of Zhu et al [11]. The shaded area is given by $T_{\text {eq }}$ calculated with the maximum and minimum values of $R_{\text {Hsh }}$ using equation (20) and (3).

the intrinsic charge carrier concentration can be calculated by [11]

$$
c_{0}=\kappa \frac{\pi^{2}}{6} k_{\mathrm{B}}^{2}\left(\frac{3 \Delta^{2}}{\pi^{2} k_{\mathrm{B}}^{2}}+T_{\text {real }}^{2}\right)
$$

where $2 \Delta$ is the width of the distribution. This formula motivates the dashed fitting line in figure 10, where

$$
T_{\text {eq }}=\sqrt{T_{\text {inh }}^{2}+T_{\text {real }}^{2}}
$$

was employed to relate $T_{\text {eq }}$ with $T_{\text {real }}$. The term $T_{\text {inh }}$ denotes the component of $T_{\text {eq }}$ that is solely induced by SCI. While equation (25) reflects qualitatively the relationship very well, better results are obtained with the heuristic formula

$$
T_{\text {eq }}=\sqrt{\alpha^{2} T_{\text {inh }}^{2}+T_{\text {real }}^{2}}-(\alpha-1) T_{\text {inh }}
$$

displayed as solid red line in figure 10 with an value of $\alpha=1.28$. Measurements of devices with different $T_{\text {inh }}$ would be necessary to judge about the general applicability of equation (26).

\section{Conclusion}

We have presented a method to calculate the surface potential of graphene as a function of the applied gate voltage taking finite temperatures into account. While the calculation have to be performed numerically, it was possible to avoid any further approximation. Once the surface potential is computed many important quantities become accessible, such as the charge carrier concentration, the sheet Hall coefficient and the total device capacitance, all as a function of the gate voltage. The presented method can be extended to account for interface traps. This was demonstrated utilizing a constant interface trap 
density, but any spectral form of $D_{\text {it }}$ can be used as long as its integral can be evaluated, may it be analytically or numerically.

A detailed discussion of the gate voltage dependent inverse sheet Hall coefficient explains the general form of $R_{\mathrm{Hsh}}^{-1}\left(V_{\mathrm{GS}}\right)$ and the impact of temperature, insulator capacitance and interface traps. This enables the determination of the intrinsic charge carrier concentration without difficult algebra. In that way we could obtain the interface trap density and the Drude mobility.

Furthermore we proposed to utilize the temperature dependence of the sheet Hall coefficient to take spatial charge inhomogeneities into account by introducing the equivalent temperature. This was demonstrated on the basis of actual measured Hall data.

\section{Acknowledgments}

The authors thank Jürgen Ristein, Lothar Ley, and Alexander Tzalenchuk for discussions. The research leading to these results has received partial funding from the European Union Seventh Framework Programme under grant agreement n ${ }^{\circ} 257829$ ConceptGraphene and grant agreement $\mathrm{n}^{\circ} 604391$ Graphene Flagship, and from the German Research Foundation (DFG) within project SE1087/7-1.

\section{References}

[1] Novoselov K S, Geim A K, Morozov S V, Jiang D, Zhang Y, Dubonos S V, Grigorieva I V and Firsov A A 2004 Electric field effect in atomically thin carbon films Science 306 666-669

[2] Schwierz F 2010 Graphene transistors Nature Nanotechnol. 5 487-496

[3] Moon J, Curtis D, Hu M, Wong D, McGuire C, Campbell P, Jernigan G, Tedesco J, VanMil B, Myers-Ward R, Eddy C and Gaskill D 2009 Epitaxial-graphene RF field-effect transistors on Si-face 6H-SiC substrates IEEE Electron Device Lett. 30650

[4] Lin Y M, Dimitrakopoulos Ch, Jenkins K, Farmer D, Chiu H Y, Grill A and Avouris Ph 2010 100-GHz transistors from wafer-scale epitaxial graphene Science $\mathbf{3 2 7} 662$

[5] Wu Y, Jenkins K A, Valdes-Garcia A, Farmer D B, Zhu Y, Bol A A, Dimitrakopoulos Ch, Zhu W, Xia F, Avouris Ph and Lin Y M 2012 State-of-the-art graphene high-frequency electronics Nano Lett. 12 3062-3067

[6] Guo Z, Dong R, Chakraborty P S, Lourenco N, Palmer J, Hu Y, Ruan M, Hankinson J, Kunc J, Cressler J D, Berger C and de Heer W A 2013 Record maximum oscillation frequency in C-face epitaxial graphene transistors Nano Lett. 13 942-947

[7] Sze S 1981 Physics of Semiconductor Devices (John Wiley and Sons (WIE))

[8] Schroder D K 2006 Semiconductor Material and Device Characterization (Wiley-IEEE Press)

[9] Fang T, Konar A, Xing H and Jena D 2007 Carrier statistics and quantum capacitance of graphene sheets and ribbons Appl. Phys. Lett. 91092109

[10] Ristein J, Zhang W, Speck F, Ostler M, Ley L and Seyller Th 2010 Characteristics of solution gated field effect transistors on the basis of epitaxial graphene on silicon carbide J. Phys. D: Appl. Phys. 43345303

[11] Zhu W, Perebeinos V, Freitag M and Avouris Ph 2009 Carrier scattering, mobilities, and electrostatic potential in monolayer, bilayer, and trilayer graphene Phys. Rev. B 80(23) 235402

[12] Takase K, Tanabe S, Sasaki S, Hibino H and Muraki K 2012 Impact of graphene quantum capacitance on transport spectroscopy Phys. Rev. B $\mathbf{8 6} 165435$ 
[13] Meric I, Han M Y, Young A F, Ozyilmaz B, Kim Ph and Shepard K L 2008 Current saturation in zero-bandgap, top-gated graphene field-effect transistors Nature Nanotechnol. 3 654-659

[14] Thiele S A, Schaefer J A and Schwierz F 2010 Modeling of graphene metal-oxide-semiconductor field-effect transistors with gapless large-area graphene channels J. Appl. Phys. 107094505

[15] Champlain J G 2011 A first principles theoretical examination of graphene-based field effect transistors J. Appl. Phys. 109084515

[16] Castro Neto A H, Peres N M R, Novoselov K S and Geim A K 2009 The electronic properties of graphene Rev. Mod. Phys. 81 109-162

[17] Hwang E and Das Sarma S 2009 Screening-induced temperature-dependent transport in twodimensional graphene Phys. Rev. B $\mathbf{7 9} 165404$

[18] Ohta T, Bostwick A, McChesney J, Seyller Th, Horn K and Rotenberg E 2007 Interlayer interaction and electronic screening in multilayer graphene investigated with angle-resolved photoemission spectroscopy Phys. Rev. Lett. 98(20) 206802

[19] Wagner T, Köhler D, Milde P and Eng L M 2013 Probing the local surface potential and quantum capacitance in single and multi-layer graphene Appl. Phys. Lett. 103023102

[20] Xia J, Chen F, Li J and Tao N 2009 Measurement of the quantum capacitance of graphene Nature Nanotechnol. 4 505-509

[21] Novoselov K S, Geim A K, Morozov S V, Jiang D, Katsnelson M I, Grigorieva I V, Dubonos S V and Firsov A A 2005 Two-dimensional gas of massless Dirac fermions in graphene Nature 438 197-200

[22] Zhang Y, Tan Y W, Stormer H L and Kim Ph 2005 Experimental observation of the quantum Hall effect and Berry's phase in graphene Nature 438 201-204

[23] Kopylov S, Tzalenchuk A, Kubatkin S and Fal'ko V I 2010 Charge transfer between epitaxial graphene and silicon carbide Appl. Phys. Lett. 97112109

[24] Deal B E 1980 Standardized terminology for oxide charges associated with thermally oxidized silicon IEEE Trans. Electron Dev. 27 606-608

[25] Martin J, Akerman N, Ulbricht G, Lohmann T, Smet J H, von Klitzing K and Yacoby A 2008 Observation of electron-hole puddles in graphene using a scanning single-electron transistor Nature Phys. 4 144-148

[26] Morozov S V, Novoselov K S, Katsnelson M I, Schedin F, Ponomarenko L A, Jiang D and Geim A K 2006 Strong suppression of weak localization in graphene Phys. Rev. Lett. 97016801

[27] Geim A K and Novoselov K S 2007 The rise of graphene Nature Mater. 6 183-191

[28] Gibertini M, Tomadin A, Guinea F, Katsnelson M I and Polini M 2012 Electron-hole puddles in the absence of charged impurities Phys. Rev. B 85 201405(R)

[29] Zhang Y, Brar V W, Girit C, Zettl A and Crommie M F 2009 Origin of spatial charge inhomogeneity in graphene Nature Phys. 5 722-726

[30] Deshpande A, Bao W, Miao F, Lau C and LeRoy B 2009 Spatially resolved spectroscopy of monolayer graphene on $\mathrm{SiO}_{2}$ Phys. Rev. B 79205411

[31] Nagashio K, Nishimura T and Toriumi A 2013 Estimation of residual carrier density near the Dirac point in graphene through quantum capacitance measurement Appl. Phys. Lett. 102173507

[32] Wehrfritz P, Fromm F, Malzer S and Seyller Th 2014 Quasi-freestanding epitaxial graphene transistor with silicon nitride top gate J. Phys. D: Appl. Phys. 47305103 


\title{
Supplementary information \\ The Hall Coefficient: A Tool For Characterizing Graphene Field Effect Transistors
}

\author{
Peter Wehrfritz and Thomas Seyller \\ Institut für Physik, Technische Universität Chemnitz, Reichenhainer Str. 70, 09126 Chemnitz, Germany \\ E-mail: thomas.seyller@physik.tu-chemnitz.de
}

The following code listing shows a sample implementation for Igor Pro 6 of the most important formulas discussed in the main paper. The functions net_charge (), carrier_conc (), and rhsh () calculate the net charge density $Q_{\mathrm{N}}$, the charge carrier concentration $c$, and the sheet Hall coefficient $R_{\mathrm{Hsh}}$, respectively, for a given value of the surface potential $\psi_{\mathrm{s}}$. With the function surfpot_from_VGS () it is possible to map the applied gate voltage $V_{\mathrm{GS}}$ to the surface potential. Finally, the function rhsh_fit () offers a fitting function that can be used with the usual Igor Pro fitting routine ( FuncFit). Note, however, that the errors of the results, due to the implicit calculation with FindRoots, are not small enough for the standard fitting settings. This can be circumvented by using a so-called epsilon wave with the entry of $1.0 \times 10^{-5}$ for all parameters. More details on the functionality and the usage of the epsilon wave can be found in the manual of Igor Pro.

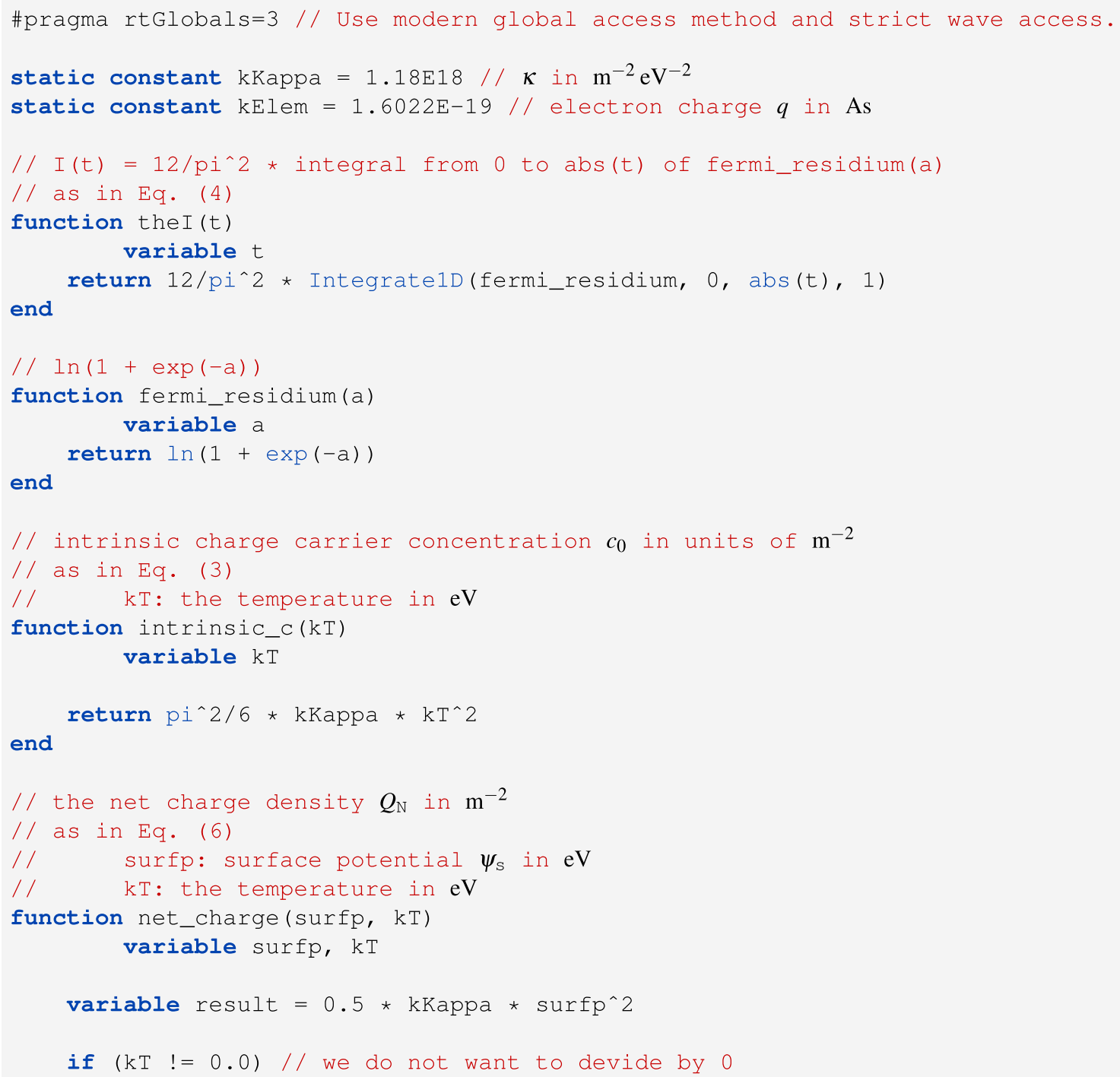




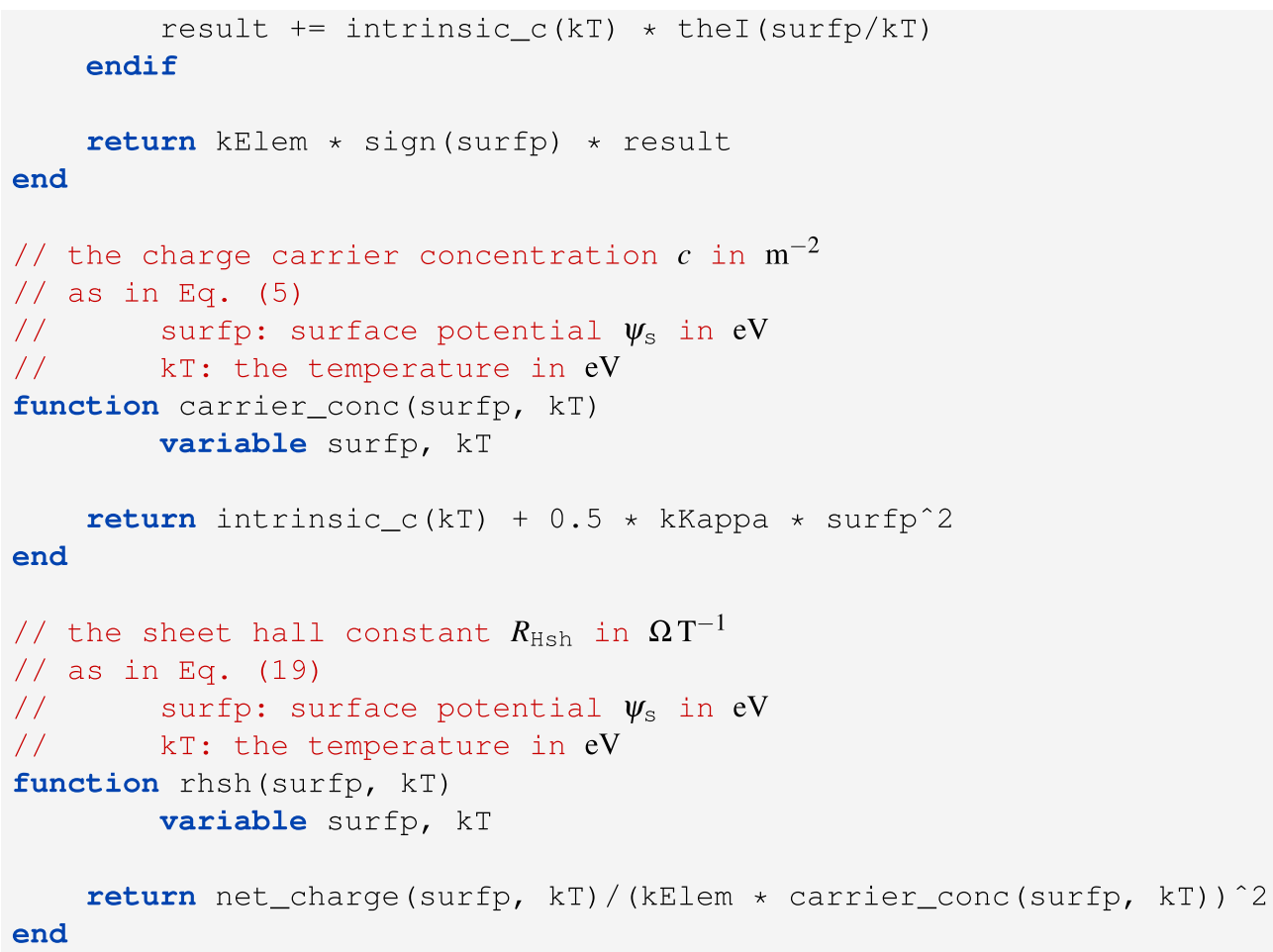




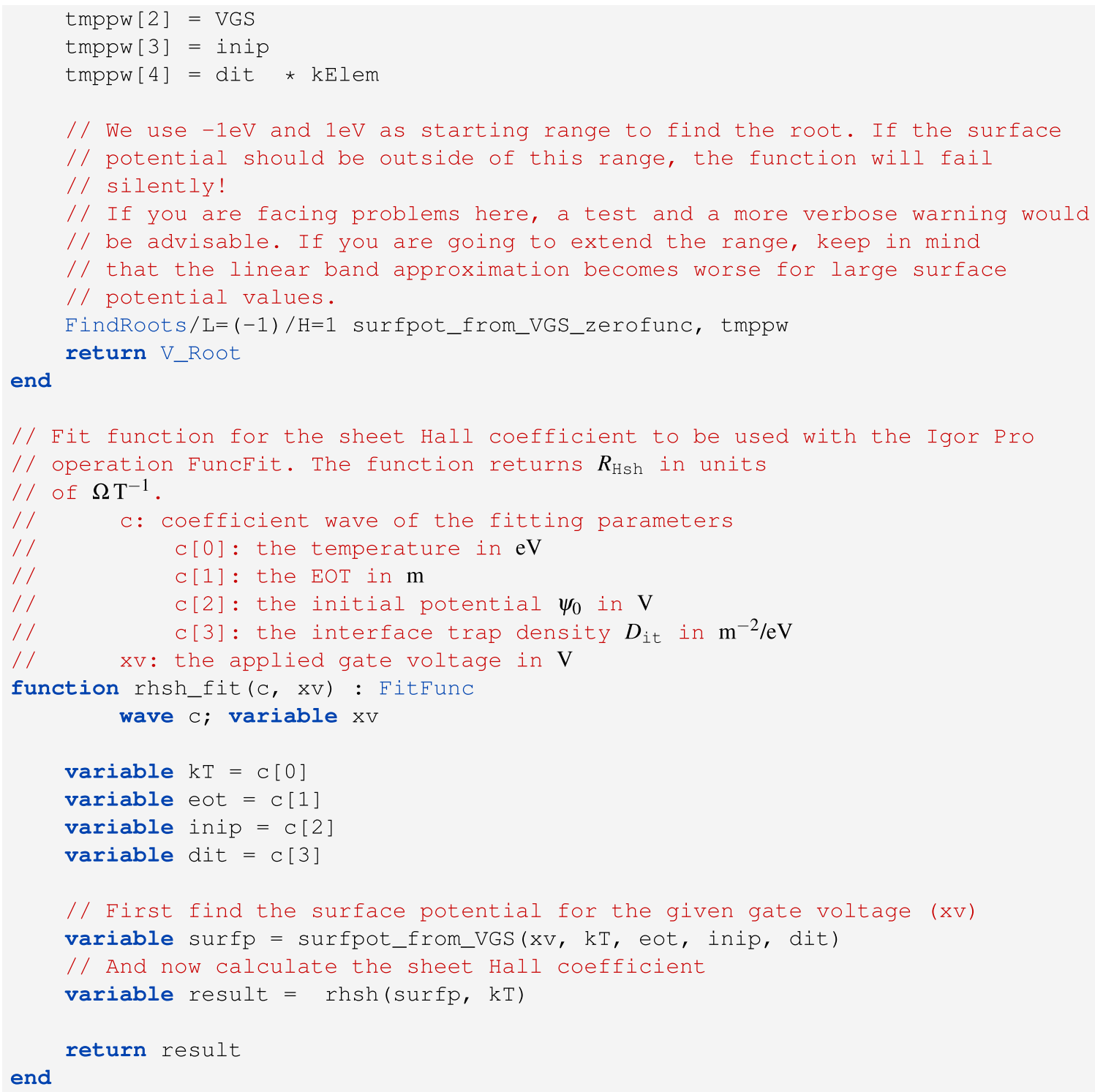

